\section{ORIGINAL RESEARCH}

T. Hattori

T. Yuasa

S. Aoki

R. Sato

H. Sawaura

T. Mori

H. Mizusawa

\title{
Altered Microstructure in Corticospinal Tract in Idiopathic Normal Pressure Hydrocephalus: Comparison with Alzheimer Disease and Parkinson Disease with Dementia
}

BACKGROUND AND PURPOSE: Previous neuropathologic studies in chronic hydrocephalus have suggested the presence of white matter damage, presumably from mechanical pressure due to ventricular enlargement and metabolic derangement. This study aimed to investigate the diffusional properties of the CST in patients with iNPH by using DTI and to determine whether this method could be used as a new diagnostic tool to differentiate patients with iNPH from those with AD and PDD and control subjects.

MATERIALS AND METHODS: We enrolled 18 patients with iNPH, 11 patients with AD, 11 patients with PDD, and 19 healthy control subjects. Diffusion tensor metrics of the segmented CST, including FA values, axial eigenvalues, and radial eigenvalues, were evaluated by using tract-specific analysis. The anisotropy color-coding tractography of the CST was visually evaluated. The DTI findings were compared among groups.

RESULTS: Tract-specific analysis of the CST showed that FA values and axial eigenvalues were significantly increased $(P<.001)$, whereas radial eigenvalues were not significantly altered, in patients with iNPH compared with other subjects. The CST tractographic images in patients with iNPH was visually different from those in other subjects $(P<.001)$. In discriminating patients with iNPH from other subjects, the CST FA values had a sensitivity of $94 \%$ and specificity of $80 \%$ at a cutoff value of 0.59 .

CONCLUSIONS: Our results suggest that patients with iNPH have altered microstructures in the CST. Quantitative and visual CST evaluation by using DTI may be useful for differentiating patients with iNPH from patients with AD or PDD or healthy subjects.

\begin{abstract}
ABBREVIATIONS: $\mathrm{AD}=$ Alzheimer disease; $\mathrm{CST}=$ corticospinal tract; $\mathrm{DTI}=$ diffusion tensor imaging; $F A=$ fractional anisotropy; $\mathrm{iNPH}=$ idiopathic normal pressure hydrocephalus; $\mathrm{MMSE}=$ Mini-Mental State Examination; PD = Parkinson disease; PDD = Parkinson disease with dementia; $\mathrm{ROI}=$ region of interest; SLF $=$ superior longitudinal fasciculus
\end{abstract}

i

NPH is a syndrome characterized by the triad of gait disturbance, urinary incontinence, and cognitive decline in the absence of elevated CSF pressure. ${ }^{1}$ Typical features of iNPH on MR imaging studies include ventricular enlargement, diminution of high-convexity sulci, and periventricular hyperintensity. ${ }^{1,2}$ Previous neuropathologic studies of chronic hydrocephalus have suggested the presence of white matter

Received January 11, 2011; accepted after revision January 24.

From the Department of Neurology and Neurological Sciences (T.H., H.M.), Graduate School, Tokyo Medical and Dental University, Tokyo, Japan; Department of Radiology (T.H., S.A., R.S.), Juntendo University, Tokyo, Japan; Departments of Neurology (T.H., T.Y., T.M.) and Neurosurgery (H.S.), Kamagaya General Hospital, Chiba, Japan; Department of Neurology (T.H.), Kanto Central Hospital, Tokyo, Japan; and Department of Radiological Sciences (R.S.), Graduate School of Human Health Sciences, Tokyo Metropolitan University, Tokyo, Japan.

This study was supported (including provision of equipment or materials) by grants received in the 2009 fiscal year from Tanabe Mitsubishi Co., Novartis Co., Boelingerlngerheim Co., Takeda Chemical Industries, Eisai Co., Dainipponsumitomo Co., AstraZeneca Co., Pfizer Co., Asteras Co., GlaxoSmitKline Co., Kissei Co., Sanofi Aventis Co., Teijin Phama Co., FP Co., Banyu Pharmaceutical Co., Otsuka Pharmaceutical Co., Japan Mediphysics Co. and Tsumura Co.

Please address correspondence to Hidehiro Mizusawa, MD, PhD, Department of Neurology and Neurological Sciences, Graduate School, Tokyo Medical and Dental University, 1-5-45 Yushima, Bunkyo-ku, Tokyo, 113-8519, Japan; e-mail: h-mizusawa.nuro@tmd.ac.jp

Indicates open access to non-subscribers at www.ajnr.org

http://dx.doi.org/10.3174/ajnr.A2570 damage, ${ }^{3-5}$ presumably resulting from mechanical pressure due to ventricular enlargement ${ }^{6}$ and metabolic derangement. ${ }^{7}$

Gait disturbance is the most frequent and reversible symptom in iNPH patients. ${ }^{8}$ Although the origin of gait disturbance is not entirely understood, various hypotheses, including deformation of the CST, have been proposed. ${ }^{1}$ The CST connects the motor cortex and spinal cord via the cerebral peduncle. It runs along the side of the lateral ventricle and is presumably deformed by ventricular enlargement. Therefore, we hypothesized that the microstructure of the CST in iNPH patients is altered by mechanical pressure resulting from ventricular enlargement.

Recently, DTI has been used frequently to evaluate white matter alteration in various neurodegenerative diseases. ${ }^{9,10}$ Diffusion anisotropy is most commonly quantified by the FA value. ${ }^{11} \mathrm{FA}$ values are calculated from the axial and radial diffusivity of water molecules. ${ }^{12}$ Axial diffusivity is defined by the axial eigenvalue ( $\lambda 1$ value), ie, the mean magnitude of water diffusion parallel to the axon. Radial diffusivity is defined by the radial eigenvalue (average value of $\lambda 2$ and $\lambda 3$ ), ie, the mean magnitude of water diffusion perpendicular to the axon. Tract-specific analysis is 1 method of DTI data analysis. It uses diffusion tensor tractography as an anatomic landmark, and diffusion tensor metrics are calculated along the drawn tractographic image. The CST is 1 of the targets most frequently evaluated by using tract-specific analysis in various diseases. ${ }^{13,14}$

From a clinical viewpoint, iNPH is considered a reversible 
cause of dementia and gait disturbance in older adults because it can be treated by shunt surgery. However, it is often difficult to make a diagnosis of iNPH. Although it is a rare disease, iNPH needs to be differentiated from other common diseases causing dementia, gait disturbance, or both. In terms of dementia, iNPH should be differentiated from $\mathrm{AD}$, the most common cause of dementia. Patients with late-phase $\mathrm{AD}$ have diffuse cerebral atrophy, leading to secondary ventricular enlargement. ${ }^{15}$ In terms of gait disturbance, iNPH should be differentiated from PD. Whereas the gait of patients with iNPH is typically described as shuffling, "magnetic," and wide-based, ${ }^{8}$ it sometimes resembles that of patients with PD, who have a stooped posture and take small shuffling steps with a freezing gait. ${ }^{16}$ In addition, patients with $\mathrm{PD}$ have a high risk of developing dementia, ${ }^{17}$ and patients with PDD also have diffuse cerebral atrophy, leading to secondary ventricular enlargement. ${ }^{18}$ Therefore, biologic markers that can differentiate iNPH from AD or PDD are needed.

Here, we aimed to elucidate the diffusional properties of the CST in patients with iNPH by using tract-specific analysis and to determine whether this method could be used as a new diagnostic tool to differentiate patients with iNPH from those with $\mathrm{AD}$ and $\mathrm{PDD}$ and healthy control subjects.

\section{Materials and Methods}

\section{Subject Selection}

Subjects were prospectively recruited from patients treated at Kamagaya General Hospital in Chiba, Japan. All subjects were medically interviewed and were given general and neurologic examinations, including the MMSE, by neurologists (T.H. and T.Y.). Information on complaints of memory loss or other subjective cognitive deficits was gathered by means of a caregiver-based interview for all patients.

Inclusion criteria were defined as follows: $\mathrm{iNPH}$ was diagnosed according to the clinical diagnostic criteria of probable iNPH. ${ }^{1} \mathrm{AD}$ was diagnosed according to the National Institute of Neurologic and Communicative Disorders and Stroke and the $\mathrm{AD}$ and Related Diseases Association criteria for a diagnosis of probable AD. ${ }^{19} \mathrm{PD}$ was diagnosed according to the clinical diagnostic criteria of the Parkinson's Disease United Kingdom Brain Bank. ${ }^{20}$ PDD was diagnosed according to the clinical diagnosis criteria for probable PDD. ${ }^{17}$ Normal control subjects were selected on the basis of a normal result in the MMSE and a normal neurologic examination.

Exclusion criteria were defined as follows: Subjects with a history of neurologic diseases such as stroke and epilepsy or with underlying diseases that potentially affected the brain (eg, uncontrolled hypertension or chronic kidney disease) were excluded.

In addition, subjects who had an Evans index between 0.3 (according to the diagnostic criteria for $\mathrm{iNPH}^{1}$ ) and the maximum Evans index score in non-iNPH subjects (ie, patients with AD or PDD and control subjects) were selected and classified into subgroups. We thus compared the noniNPH subgroups with the iNPH subgroup under conditions in which all subjects had similar extents of ventricular enlargement.

This study was approved by the institutional review board of Kamagaya General Hospital, and written informed consent was obtained from all subjects.

\section{Staging of Leukoaraiosis}

The extent of periventricular white matter T2 hyperintensities (ie, leukoaraiosis) was rated for all subjects according to the Fazekas classification. $^{21}$

\section{DTI Data Acquisition}

DTI data were obtained with a 1.5T clinical scanner (Signa Excite; GE Healthcare, Milwaukee, Wisconsin) with a single-shot echo-planar sequence $\left(\mathrm{TR}=14,000 \mathrm{~ms}\right.$, TE $=103.5 \mathrm{~ms}, b=1000 \mathrm{~s} / \mathrm{mm}^{2}, 13$ different axes of motion-probing gradients, FOV $=280 \mathrm{~mm}$, matrix $=128 \times 128$ [interpolated image matrix size, $256 \times 256$ ], section spacing $=3 \mathrm{~mm}$, averaging $=2$, number of sections $=50$, and total acquisition time $=7$ minutes).

\section{Tractography and Tract-Specific Analysis of CST}

DTI data were analyzed by using dTV II and VOLUME-ONE 1.72, developed by Masutani et $\mathrm{al}^{11}$ (http://www.ut-radiology.umin.jp/ people/masutani/dTV.htm) and running on Windows XP Professional (Microsoft, Redmond, Washington). The threshold of linetracking was set at FA $>0.18$. Diffusion tensor tractography of the CST was generated by using a 2-ROI method, with the seed ROI on the motor cortex and the target ROI on the cerebral peduncle (Fig $1 A$, $-B)$. The CST crosses the SLF at the level of the corona radiata. ${ }^{22}$ The SLF is identified as the green areas next to the CST on a color-coded FA map of the coronal section in which the CST crosses the SLF (Fig $1 C)$. As an anatomic landmark for the CST, we used the uppermost part of the SLF instead of the lateral ventricle used in a previous study, ${ }^{13}$ on the basis of the assumption that the areas where the CST and the SLF cross are not affected by the presence or absence of ventricular enlargement (Fig 1). Voxelization along the CST was performed from the uppermost part of the SLF to the posterior limb of the internal capsule by using dTV (Fig 1). The level of the posterior limb of the internal capsule also was identified at one-third of the height from the cerebral peduncle to the uppermost part of the SLF. We evaluated this area in the CST because this area of tractography of the CST was stably drawn. The mean values of the diffusion tensor metrics (ie, FA values, axial eigenvalues, and radial eigenvalues) were measured in the voxelized area of tractography of the CST by using tract-specific analysis, and were averaged between the right and left hemispheres.

The tractographic colors were visualized by diffusion anisotropy coded from yellow (low anisotropy) to orange (high anisotropy). ${ }^{11}$ We visually evaluated the same segment of the CST in a tract-specific analysis (Fig $2 A,-B$ ). To avoid any bias resulting from knowledge of ventricular enlargement, the lower and upper parts of the tractography and images of the brain and CSF were not used for visual evaluation. The visual tractographic features of the CST were classified as "type A" (low anisotropy and rough) or "type B" (high anisotropy and straight; Fig $2 C,-D$ ) by a neurologist (T.H.) and a neuroradiologist (S.A.) blinded to the clinical information.

When creating the tractographic image, the operator (R.S.) was blinded to the clinical information on each subject to avoid bias.

\section{Statistical Analysis}

Statistical analysis among groups was performed by using analysis of variance with the post hoc Tukey honestly significant difference test for continuous variables, the Kruskal-Wallis test with post hoc MannWhitney $U$ tests for noncontinuous variables, and the $\chi^{2}$ test for categorical data. The tractographic features (the proportion of type B) were compared among groups by using the $\chi^{2}$ test with a post hoc Pearson $\chi^{2}$ test. The criterion of statistical significance was $P<.05$.

Receiver operating characteristic analysis was used to calculate the optimal cutoffs of the FA value and axial eigenvalue in the CST for patients with iNPH to differentiate their tracts from those of patients with AD or PDD or of control subjects. Analyses were performed with 

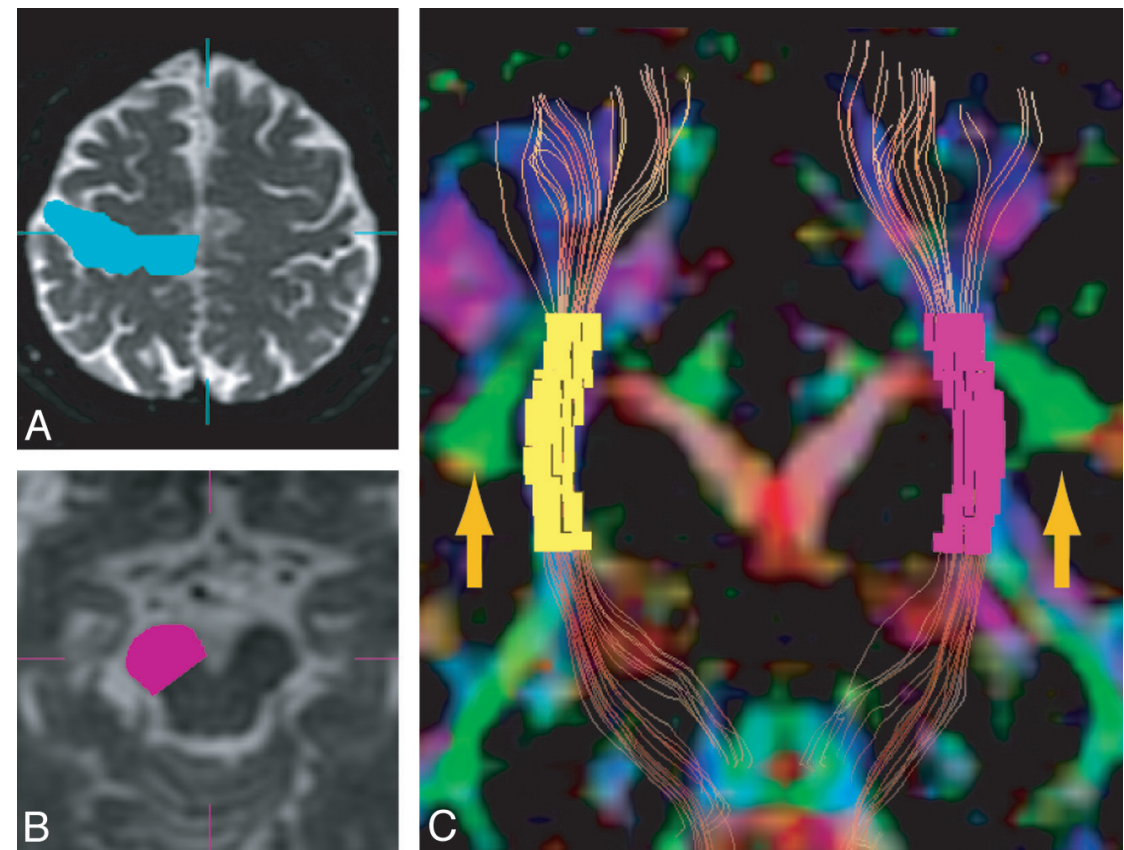

Fig 1. Voxelization of part of the CST by tract-specific analysis. Tractographic images of the CST were generated by using the 2-ROI method of seed ROI in the motor cortex (A) and target $\mathrm{ROI}$ in the cerebral peduncle $(B)$. C. Tractography of the CST is shown with a color-coded FA map (coronal section). Voxelization along the CST was performed between the uppermost part of the SLF (green area, arrow) and the posterior limb of the internal capsule by using dTV software.
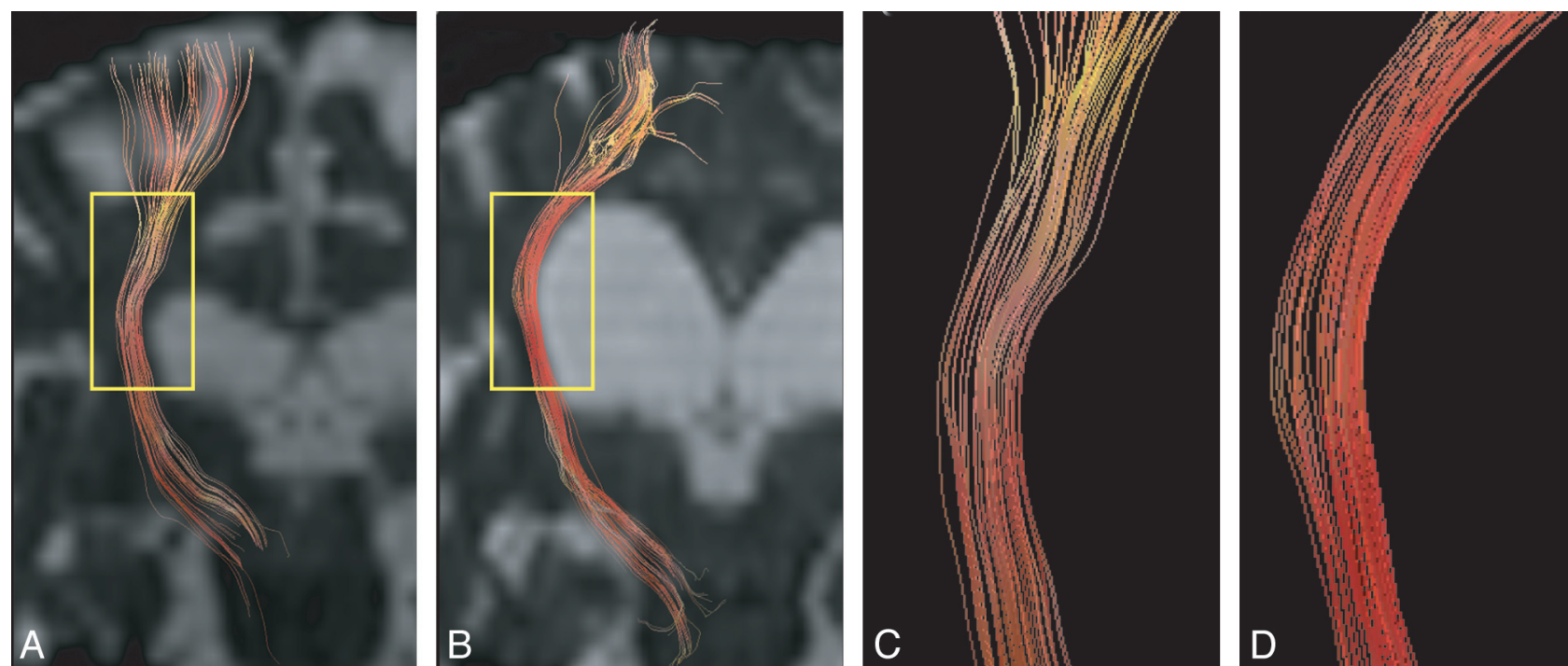

Fig 2. Visual evaluation of anisotropy color-coding tractography of the CST. Anisotropy color-coding tractography of the CST in a control subject (A) and a patient with iNPH (B) is shown, with $b=0$ images. The areas defined by the yellow squares in $A$ and $B$ were used for visual evaluation in $C$ and $D$. $C$, Type $\mathrm{A}$ (low anisotropy and rough). $D$, Type $B$ (high anisotropy and straight).

Scientific Package for Social Sciences, Version 11 (SPSS, Chicago, Illinois). The results are expressed as means \pm SD.

\section{Results}

\section{Demographic and Clinical Features}

On the basis of the above-mentioned inclusion and exclusion criteria, we enrolled 18 patients with iNPH, 11 patients with $\mathrm{AD}$, and 11 patients with PDD, as well as 19 age- and sexmatched control subjects (Table 1). All patients with iNPH had cerebral ventricular enlargement (Evans index $>0.3$ ) and narrowed CSF spaces at high convexity on MR imaging. The demographic and clinical features of patients with iNPH, AD, or PDD and of control subjects are listed in Table 1. The Evans index was significantly higher in patients with iNPH than in other subjects $(P<.001)$. All patient groups had significantly lower MMSE scores than control subjects $(P<.001)$. There were no significant differences in age, sex, or vascular risk factors (eg, hypertension, diabetes mellitus, and dyslipidemia) among the groups.

\section{Extent of Leukoaraiosis}

The Fazekas classifications of leukoaraiosis for all groups are listed in Table 2. The extent of leukoaraiosis was significantly greater in patients with iNPH than in patients with $\operatorname{PDD}(P=$ 


\begin{tabular}{|c|c|c|c|c|c|c|}
\hline Characteristic & $\begin{array}{l}\text { A, iNPH } \\
(n=18)\end{array}$ & $\begin{array}{c}\mathrm{B}, \mathrm{AD} \\
(n=11)\end{array}$ & $\begin{array}{c}\text { C, PDD } \\
(n=11)\end{array}$ & $\begin{array}{l}\text { D, Control } \\
(n=19)\end{array}$ & $P$ & $\begin{array}{c}\text { Post Hoc } \\
\text { Significance }\end{array}$ \\
\hline$\overline{\text { Age }(\mathrm{yr})}$ & $77.3 \pm 4.7$ & $72.1 \pm 5.5$ & $75.6 \pm 5.5$ & $75.6 \pm 5.5$ & .222 & \\
\hline Sex (M:F) & $7: 11$ & $5: 6$ & $6: 5$ & $7: 12$ & .805 & \\
\hline MMSE score & $22.1 \pm 3.7$ & $18.3 \pm 6.7$ & $22.4 \pm 2.7$ & $28.6 \pm 0.0$ & $<.001$ & $A, B, C<D^{a}$ \\
\hline Evans index & $0.37 \pm 0.04$ & $0.29 \pm 0.03$ & $0.29 \pm 0.04$ & $0.28 \pm 0.03$ & $<.001$ & $A>B, C, D^{a}$ \\
\hline \multicolumn{7}{|l|}{ Vascular risk factor } \\
\hline Hypertension (\%) & 27.8 & 36.4 & 18.2 & 42.1 & .561 & \\
\hline Diabetes mellitus (\%) & 16.7 & 18.2 & 0.0 & 0 & .157 & \\
\hline Dyslipidemia (\%) & 22.2 & 27.3 & 18.2 & 0 & .168 & \\
\hline
\end{tabular}

Note:-Data are presented as means \pm SD

a $P<.001$.

Table 2: Tract-specific analysis and visual evaluation of tractography of the CST

\begin{tabular}{|c|c|c|c|c|c|c|}
\hline & $\begin{array}{l}\text { A, iNPH } \\
(n=18)\end{array}$ & $\begin{array}{c}\mathrm{B}, \mathrm{AD} \\
(n=11)\end{array}$ & $\begin{array}{c}\mathrm{C}, \mathrm{PDD} \\
(n=11)\end{array}$ & $\begin{array}{l}\text { D, Control } \\
(n=19)\end{array}$ & $P$ & $\begin{array}{c}\text { Post Hoc } \\
\text { Significance }\end{array}$ \\
\hline Extent of leukoaraiosis (Fazekas classification) & $2.06 \pm 0.87$ & $1.64 \pm 0.92$ & $1.27 \pm 0.90$ & $0.89 \pm 0.81$ & .003 & $A>C, D^{a}$ \\
\hline \multicolumn{7}{|l|}{ Tract-specific analysis } \\
\hline FA value & $0.62 \pm 0.03$ & $0.54 \pm 0.04$ & $0.55 \pm 0.04$ & $0.55 \pm 0.04$ & $<.001$ & $A>B, C, D^{b}$ \\
\hline Axial eigenvalue & $1.65 \pm 0.10$ & $1.49 \pm 0.07$ & $1.42 \pm 0.08$ & $1.43 \pm 0.07$ & $<.001$ & $A>B, C, D^{b}$ \\
\hline Radial eigenvalue & $0.53 \pm 0.08$ & $0.56 \pm 0.05$ & $0.54 \pm 0.05$ & $0.54 \pm 0.04$ & .422 & \\
\hline \multicolumn{7}{|l|}{ Visual evaluation of tractography } \\
\hline Pattern $\mathrm{B}, n(\%)$ & $17(94.4)$ & $3(27.2)$ & $1(9.1)$ & $1(5.3)$ & $<.001$ & $A>B, C, D^{b}$ \\
\hline
\end{tabular}

Note:-Data are presented as means \pm SD.

a $P<.05$.

b $P<.001$

\begin{tabular}{|c|c|c|c|c|c|c|}
\hline Variable & Sensitivity & Specificity & $\begin{array}{c}\text { Positive } \\
\text { Predictive Value }\end{array}$ & $\begin{array}{c}\text { Negative } \\
\text { Predictive Value }\end{array}$ & $\begin{array}{l}\text { Cutoff Value of } \\
\text { Each Measure }\end{array}$ & $\begin{array}{c}\text { Area } \\
\text { Under } \\
\text { Curve }\end{array}$ \\
\hline \multicolumn{7}{|l|}{ FA value } \\
\hline Compared with $A D$ & 94 & 82 & 89 & 90 & 0.59 & 0.92 \\
\hline Compared with PDD & 94 & 72 & 85 & 89 & 0.59 & 0.89 \\
\hline Compared with control & 94 & 84 & 85 & 94 & 0.59 & 0.91 \\
\hline Compared with AD, PDD, and control & 94 & 80 & 68 & 97 & 0.59 & 0.91 \\
\hline \multicolumn{7}{|l|}{ Axial eigenvalue } \\
\hline Compared with $A D$ & 94 & 82 & 89 & 90 & 1.52 & 0.95 \\
\hline Compared with PDD & 100 & 82 & 90 & 100 & 1.48 & 0.96 \\
\hline Compared with control & 100 & 84 & 86 & 100 & 1.50 & 0.98 \\
\hline Compared with $\mathrm{AD}, \mathrm{PDD}$, and control & 94 & 83 & 71 & 97 & 1.52 & 0.97 \\
\hline
\end{tabular}

Note:-Data (except for the cutoffs of FA values and axial eigenvalues) are shown as percentages. Receiver operating characteristic analysis was used to determine the optimum cutoff values for evaluating the usefulness of FA values and axial eigenvalues in differentiating iNPH from AD, PDD, and controls.

$.035)$ or control subjects $(P=.01)$. The extent of leukoaraiosis was not significantly different between patients with iNPH and patients with $\mathrm{AD}(P=.238)$.

\section{Diffusion Tensor Metrics in CST of Patients with iNPH, $A D$, or PDD and in Control Subjects}

The diffusion tensor metrics in the CST, as measured by using tract-specific analysis, are listed in Table 2. FA values $(P<.001)$ and axial eigenvalues $(P<.001)$ were significantly increased in patients with iNPH, whereas radial eigenvalues were not significantly altered, compared with those in other subjects.

Table 3 summarizes the diagnostic discrimination of iNPH subjects from the other groups by using FA values and axial eigenvalues of the CST.

\section{Visual Evaluation of Tractographic Images of CST}

Visual evaluation of the tractographic images of the CST showed a greater proportion of type B in patients with iNPH
(94.4\%) than in patients with $\mathrm{AD}(27.2 \%)$ and PDD (9.1\%), or in control subjects (5.3\%; Table 2$)$. The proportion of type $\mathrm{B}$ was significantly higher in patients with iNPH than in patients with $\mathrm{AD}$ or $\mathrm{PDD}$ or in control subjects $(P<.001)$. There was sufficient agreement between the assessments made of the tractographic pattern by 2 raters (agreement rate, 52 [88.1\%] of 59 subjects) and between 2 assessments made by 1 rater with a 3-month interval between ratings (agreement rate, 54 [91.5\%] of 59 subjects).

\section{Comparison of Non-iNPH and iNPH Subjects with Similar Extents of Ventricular Enlargement}

The maximum Evans index of our non-iNPH subjects was 0.35. Therefore, subjects who had Evans indexes within the range of 0.3 to 0.35 (ie, 7 patients with iNPH, 4 patients with $\mathrm{AD}, 6$ patients with PDD, and 6 control subjects) were enrolled in this part of the study. There were no significant differences in age, sex, vascular risk factors, Evans index (iNPH, $0.32 \pm 0.02 ; \mathrm{AD}, 0.33 \pm 0.02 ; \mathrm{PDD}, 0.32 \pm 0.02$; control, 

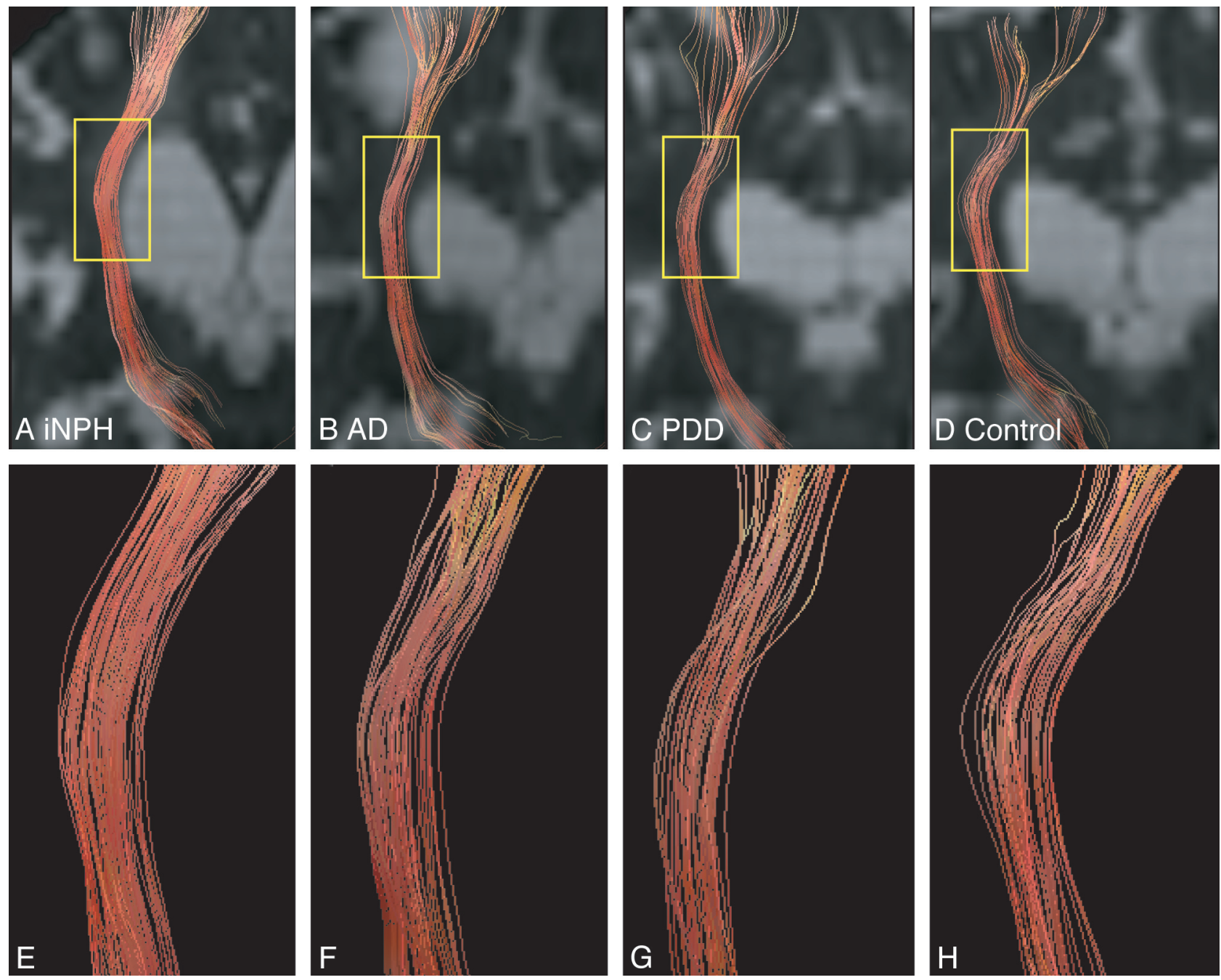

Fig 3. Examples of anisotropy color-coding tractography of the CST. Anisotropy color-coding tractography of the CST in patients with iNPH (Evans index: 0.31 ; $A)$, AD (0.33; $B)$, PDD (0.33; $C$ ), and a control subject $(0.31 ; D)$ are shown, with $b=0$ images. The areas defined by the yellow squares in $A, B, C$, and $D$ are shown in $E, F, G$, and $H$, respectively.

$0.32 \pm 0.02 ; P=.263)$, or radial eigenvalues $(\mathrm{iNPH}, 0.54 \pm$ $0.05 ; \mathrm{AD}, 0.55 \pm 0.15 ; \mathrm{PDD}, 0.52 \pm 0.02$; control, $0.55 \pm 0.04$ $P=.888)$ among all subgroups. FA values were significantly higher in patients with iNPH $(0.62 \pm 0.02)$ than in patients with $\mathrm{AD}(0.56 \pm 0.02 ; P=.009)$ or $\mathrm{PDD}(0.58 \pm 0.02$; $P=.038)$, or in control subjects $(0.55 \pm 0.04 ; P=.001)$. Axial eigenvalues were also significantly higher in patients with iNPH $(1.66 \pm 0.09)$ than in patients with $\mathrm{AD}(1.51 \pm 0.05$; $P=.025)$ or $\operatorname{PDD}(1.50 \pm 0.09 ; P=.006)$, or in control subjects $(1.50 \pm 0.04 ; P=.005)$.

Examples of tractographic images of the CST are shown in Fig 3. Visual evaluation of the tractographic images of the CST showed a greater proportion of type B in patients with iNPH (85.7\%), AD (25.0\%), and PDD (16.7\%) than in control subjects $(16.7 \%)$. The proportion of type $\mathrm{B}$ was significantly higher in patients with iNPH than in patients with $\mathrm{AD}$ $(P=.044)$ or $\mathrm{PDD}(P=.013)$ or in control subjects $(P=.013)$.

\section{Discussion}

Recently, DTI has been applied frequently to the examination of various neurologic diseases. ${ }^{9,10}$ It is a sensitive tool for detecting subtle microstructural alterations in the cerebral white matter. ${ }^{23}$ Although FA values decrease in most disease and aging processes, this study showed increased FA values in the CST of patients with iNPH. Increased FA values in the CST also were reported in a previous DTI study of iNPH that used ROI and voxel-based analysis. ${ }^{24}$ However, this is the first study that quantified the diffusion tensor metrics, including FA values and axial and radial eigenvalues, of the CST by using tractspecific analysis and visually evaluated CST tractography in patients with iNPH; our study then compared the findings with those in patients with AD or PDD and in control subjects.

In our study, patients with iNPH had significantly more extensive leukoaraiosis than patients with PDD or control subjects. In general, the leukoaraiosis indicates structural white matter damage such as myelin loss and ischemia, ${ }^{25}$ which usually decreases FA values. However, in patients with iNPH, the leukoaraiosis was distributed predominantly around the anterior horn or posterior horn of the lateral ventricle, and it was very limited in extent in the area around the segmented CST in the tract-specific analysis. Thus, we presume that there are little effects of leukoaraiosis for the segmented CST areas.

Here, tract-specific analysis showed that the FA values and axial eigenvalues in the CST were significantly increased, 
whereas radial eigenvalues were not significantly altered, in patients with iNPH compared with control subjects. In general, FA values are positively correlated with axial eigenvalues and negatively correlated with radial eigenvalues. ${ }^{12}$ Therefore, the increased FA values in part of the CST in patients with iNPH are due mainly to the increase in axial eigenvalues. As an explanation for these results, we speculate as follows: the brain parenchyma of patients with iNPH is stretched and compressed by mechanical pressure from ventricular enlargement, resulting in stretching of the neural fibers that microscopically show reduced winding and a tendency to align in the direction of stretch. In this altered microstructure, water diffusivity parallel to the axon is enhanced, leading to an increase in axial eigenvalues.

In addition, there might be another explanation for increased FA values. The CST crosses the SLF as well as interhemispheric fibers at the level of the corona radiata. ${ }^{22}$ In general, FA values decrease at points where fibers cross, ${ }^{11}$ and they increase when the crossing fibers are lost and only the fibers of interest are left. Interhemispheric fibers pass through the corpus callosum. Mechanical pressure due to ventricular enlargement stretches and compresses the corpus callosum in iNPH, ${ }^{26,27}$ and this may result in axonal loss and Wallerian degeneration of the interhemispheric fibers. In fact, one study has shown decreased FA values in the corpus callosum in patients with iNPH. ${ }^{24}$ The SLF also may be damaged from mechanical stretching, metabolic derangement, or both because the SLF runs along the side of the lateral ventricle and connects broad areas of the brain that might be damaged by the diffuse metabolic derangement in iNPH. ${ }^{7}$ Thus, decreased integrity of crossing fibers also may contribute to the increased FA values in the CST. Further studies exploring the cerebral white matter damage are needed.

Visual evaluation of tractography of the CST demonstrated that most patients with iNPH (94.4\%) had type B characteristics (high anisotropy and straight). In contrast, most patients with $\mathrm{AD}(72.8 \%)$ or PDD (90.9\%) and most control subjects (94.7\%) were of type A (low anisotropy and rough). The anisotropy, expressed as color in the tractography, followed the same trend as the FA values. Thus, the results of visual evaluation of the tractography as particular colors in patients with iNPH were consistent with the increased FA values in the tract-specific analysis. As mentioned, the CST crosses the SLF and interhemispheric fibers. ${ }^{22}$ We speculate that, in control subjects, the presence of complex microstructures in the CST, such as various crossing fibers and microscopic winding of fibers, leads to low FA values and fluctuating axial eigenvectors. These microscopic bases may underlie the macroscopic appearance of the tractography in control subjects (low anisotropy and rough). In contrast, we speculate that, in patients with iNPH, there are altered microstructures in the CST, such as stretched neural fibers with or without decreased integrity of crossing fibers and that this leads to increased FA values and less fluctuation in axial eigenvectors. These microscopic bases may underlie the macroscopic appearance of tractographic images in patients with iNPH (high anisotropy and straight).

It is often difficult to differentiate iNPH from other common diseases such as AD and PDD. CSF fluid-tap testing and external lumbar drainage testing may help to make a diagnosis of iNPH. ${ }^{28}$ However, these are invasive tests. In addition, CSF tap testing has poor sensitivity, ${ }^{29}$ and external lumbar drainage testing does not have good negative predictive values. ${ }^{8}$ Therefore, an accurate method that can identify iNPH and that preferably is noninvasive is needed. Here, we successfully differentiated patients with iNPH from patients with $\mathrm{AD}$ or PDD or control subjects by using tract-specific analysis and visual evaluation of tractography of the CST. These results also were confirmed in a comparison between non-iNPH subgroups and the iNPH subgroup under conditions in which all subjects had similar extents of ventricular enlargement. Thus, diffusion tensor metrics may be a more specific biomarker of iNPH than the Evans index, and DTI analysis of the CST may be a promising tool for differentiating iNPH from other diseases.

Visual evaluation of tractography is technically easier than performing tract-specific analysis. The dTV II software that we used here has been used in many DTI studies. ${ }^{13,30}$ In addition, the same algorithm as used in dTV II is integrated into the clinical MR imaging software of a major vendor (GE Healthcare). Therefore, visual evaluation of tractography is already applicable to clinical practice in certain environments and may be readily available as a screening tool for iNPH, followed by tract-specific quantitative evaluation.

This study, however, had several limitations. First, we included limited numbers of patients with AD or PDD who had Evans indexes $>0.3$. Further studies that include more noniNPH patients with ventricular enlargement are necessary. Second, only limited information on the pathology of iNPH, especially in regard to the CST, is available. Thus, pathologic confirmation of altered diffusion tensor metrics is needed in a future study. Third, we did not evaluate diffusion tensor metrics in patients with iNPH who had had shunt surgery. We therefore do not know the nature of the changes in diffusion tensor isometrics in patients with iNPH after shunt surgery. Additional studies including patients who have had shunt surgery are needed.

\section{Conclusions}

This study demonstrated that FA values and axial eigenvalues were significantly greater in the CST of patients with iNPH than in control subjects. These results suggest that there are altered microstructures in the CST in patients with iNPH, presumably resulting from the mechanical pressure due to ventricular enlargement with or without a decrease in the abundance of crossing fibers. By using this method, patients with iNPH were successfully differentiated from patients with AD or PDD and control subjects. The applicability of this method should be further investigated, including more patients with secondary ventricular enlargement and nonresponders to shunt surgery.

\section{Acknowledgments}

We thank Drs Atsushi Shiraishi, Department of Emergency Medicine, and Satoru Ishibashi, Department of Neurology and Neurologic Sciences, Graduate School, Tokyo Medical and Dental University, Tokyo, Japan, for discussion. We also thank Atsuhiko Hattori, Takanori Odakura, Maki Kyono, and Ryohei Abe, Department of Radiology, Kamagaya General Hospital, for cooperation. We thank the Japanese Ministry of 
Education, Culture, Sports and Technology's Comprehensive Brain Science Network for providing a good environment in which to have discussions.

Disclosures: Hidehiro Mizusawa; Speaker Bureau: Honoraria for sponsored lectures in the 2009 fiscal year: Details: Tanabemitsubishi Co.; Sanofi Aventis Co.

\section{References}

1. Relkin N, Marmarou A, Klinge P, et al. Diagnosing idiopathic normal-pressure hydrocephalus. Neurosurgery 2005;57:S4-16; discussion ii-v

2. Sasaki M, Honda S, Yuasa T, et al. Narrow CSF space at high convexity and high midline areas in idiopathic normal pressure hydrocephalus detected by axial and coronal MRI. Neuroradiology 2008;50:117-22

3. Del Bigio MR, Wilson MJ, Enno T. Chronic hydrocephalus in rats and humans: white matter loss and behavior changes. Ann Neurol 2003;53:337-46

4. Akai K, Uchigasaki S, Tanaka U, et al. Normal pressure hydrocephalus. Neuropathological study. Acta Pathol Jpn 1987;37:97-110

5. Ding Y, McAllister JP, 2nd, et al. Axonal damage associated with enlargement of ventricles during hydrocephalus: a silver impregnation study. Neurol Res 2001;23:581-87

6. Del Bigio MR. Neuropathological changes caused by hydrocephalus. Acta Neuropathol 1993;85:573-85

7. Kondziella D, Sonnewald U, Tullberg M, et al. Brain metabolism in adult chronic hydrocephalus. J Neurochem 2008;106:1515-24

8. Marmarou A, Young HF, Aygok GA, et al. Diagnosis and management of idiopathic normal-pressure hydrocephalus: a prospective study in 151 patients. J Neurosurg 2005;102:987-97

9. Sage CA, Peeters RR, Gorner A, et al. Quantitative diffusion tensor imaging in amyotrophic lateral sclerosis. Neuroimage 2007;34:486-99

10. Lee DY, Fletcher E, Martinez O, et al. Regional pattern of white matter microstructural changes in normal aging, MCI, and AD. Neurology 2009;73:1722-28

11. Masutani $\mathrm{Y}$, Aoki $\mathrm{S}$, Abe $\mathrm{O}$, et al. MR diffusion tensor imaging: recent advance and new techniques for diffusion tensor visualization. Eur J Radiol 2003;46: 53-66

12. Le Bihan D, Mangin JF, Poupon C, et al. Diffusion tensor imaging: concepts and applications. J Magn Reson Imaging 2001;13:534-46

13. Yasmin H, Aoki S, Abe O, et al. Tract-specific analysis of white matter pathways in healthy subjects: a pilot study using diffusion tensor MRI. Neuroradiology 2009;51:831-40

14. Hong YH, Sung JJ, Kim SM, et al. Diffusion tensor tractography-based analysis of the pyramidal tract in patients with amyotrophic lateral sclerosis. J Neuroimaging 2008; 18:282-87
15. Nestor SM, Rupsingh R, Borrie M, et al. Ventricular enlargement as a possible measure of Alzheimer's disease progression validated using the Alzheimer's disease neuroimaging initiative database. Brain 2008;131:2443-54

16. Jankovic J. Parkinson's disease: clinical features and diagnosis. J Neurol Neurosurg Psychiatry 2008;79:368-76

17. Emre M, Aarsland D, Brown R, et al. Clinical diagnostic criteria for dementia associated with Parkinson's disease. Mov Disord 2007;22:1689-707; quiz 837

18. Apostolova LG, Beyer M, Green AE, et al. Hippocampal, caudate, and ventricular changes in Parkinson's disease with and without dementia. Mov Disord 2010;25:687-88

19. McKhann G, Drachman D, Folstein M, et al. Clinical diagnosis of Alzheimer's disease: report of the NINCDS-ADRDA Work Group under the auspices of Department of Health and Human Services Task Force on Alzheimer's Disease. Neurology 1984;34:939-44

20. Hughes AJ, Daniel SE, Kilford L, et al. Accuracy of clinical diagnosis of idiopathic Parkinson's disease: a clinico-pathological study of 100 cases. J Neuro Neurosurg Psychiatry 1992;55:181-84

21. Fazekas F, Chawluk JB, Alavi A, et al. MR signal abnormalities at $1.5 \mathrm{~T}$ in Alzheimer's dementia and normal aging. AJR Am J Roentgenol 1987;149: 351-56

22. Wiegell MR, Larsson HB, Wedeen VJ. Fiber crossing in human brain depicted with diffusion tensor MR imaging. Radiology 2000;217:897-903

23. Dineen RA, Vilisaar J, Hlinka J, et al. Disconnection as a mechanism for cognitive dysfunction in multiple sclerosis. Brain 2009;132:239-49

24. Hattingen E, Jurcoane A, Melber J, et al. Diffusion tensor imaging in patients with adult chronic idiopathic hydrocephalus. Neurosurgery 2010;66:917-24

25. Fazekas F, Schmidt R, Scheltens P. Pathophysiologic mechanisms in the development of age-related white matter changes of the brain. Dement Geriatr Cogn Disord 1998;9 Suppl 1:2-5

26. Roricht S, Meyer BU, Woiciechowsky C, et al. Callosal and corticospinal tract function in patients with hydrocephalus: a morphometric and transcranial magnetic stimulation study. J Neurol 1998;245:280-88

27. Mataro M, Matarin M, Poca MA, et al. Functional and magnetic resonance imaging correlates of corpus callosum in normal pressure hydrocephalus before and after shunting. J Neurol Neurosurg Psychiatry 2007;78:395-98

28. Shprecher D, Schwalb J, Kurlan R. Normal pressure hydrocephalus: diagnosis and treatment. Curr Neurol Neurosci Rep 2008;8:371-76

29. Walchenbach R, Geiger E, Thomeer RT, et al. The value of temporary external lumbar CSF drainage in predicting the outcome of shunting on normal pressure hydrocephalus. J Neurol Neurosurg Psychiatry 2002;72:503-06

30. Taoka T, Kin T, Nakagawa $H$, et al. Diffusivity and diffusion anisotropy of cerebellar peduncles in cases of spinocerebellar degenerative disease. Neuroimage 2007;37:387-93 